\section{REVISTA}

MEXICANA DE

ECONOMÍA Y

FINANZAS

REMEF

(THE MEXICAN JOURNAL OF

ECONOMICS AND FINANCE)

\section{Revista Mexicana de Economía y Finanzas} Nueva Época

Volumen 15 Número 1, Enero - Marzo 2020, pp. 81-103

DOI: https://doi.org/10.21919/remef.v15i1.396

fundación de investigación

(Recibido: 7/mayo/2019, aceptado: 8/octubre/2019)

\title{
Selección del modelo de mejor estimación del Valor Razonable en un mercado emergente
}

\author{
Paula Morales Bañuelos ${ }^{1}$
}

Universidad Iberoamericana. México

\section{Resumen}

El objetivo de este trabajo es encontrar el modelo que brinde la mejor estimación posible del Valor Razonable de las partidas que componen los estados financieros, considerando la ambigüedad en la normatividad sobre el cálculo del mismo. Para ello se compararon tres metodologías: Flujos de Efectivo Descontados, Opciones Reales bajo los procesos Browniano Geométrico (MBG) y Browniano Aritmético (MBA). A pesar de que son muy pocos los estudios que han realizado este tipo de investigación, se ha llegado a la conclusión de que resulta más adecuado modelar un proceso aditivo como un MBA. Por dicha razón, se proyectaron los ingresos y posteriormente el flujo de efectivo anual de 2019 a 2028, posteriormente, se calcularon las Opciones Reales incorporando la flexibilidad de expansión y contracción de forma conjunta. De conformidad con los resultados obtenidos y en concordancia con lo sugerido por diferentes autores, el modelo que brinda la mejor aproximación posible del Valor Razonable es el de Opciones Reales bajo un MBG, con lo cual se recomienda que las empresas mexicanas conozcan y apliquen este modelo, complementando la valuación tradicional de flujos de efectivos descontados con el propósito de presentar información relevante para la toma de decisiones. Clasificación JEL: G12, G13, C4, C5, C15

Palabras clave: Flujos de Efectivo Descontados, Opciones Reales como un Movimiento Browniano Geométrico, Opciones Reales como un Movimiento Browniano Aritmético, GARCH (1,1), Método de Winters

\section{Selecting the model with the best fair value estimate in an emerging market}

\section{Abstract}

This work aims to find the model that provides the best possible estimate of the fair value of the items that comprise financial statements, considering the ambiguity in the regulations on the calculation of the same. For this purpose, three methodologies were compared: Discounted Cash Flows, Real Options under a Brownian Geometric Motion (BGM), and Real Options under an Arithmetic Brownian Motion (ABM). Although there have been few studies on this type of research, the conclusion reached is that modeling an additive process as an ABM is the most suitable. Therefore, the revenues and annual cash flow from 2019 to 2028 were projected, and, subsequently, the Real Options were calculated, incorporating expansion and contraction flexibility possibilities. The obtained results indicate that the model of Real Options under a

\footnotetext{
${ }^{1}$ Departamento de Estudios Empresariales. Universidad Iberoamericana. México. Correo electrónico: paula.morales@ibero.mx. Teléfono:+52-55- 59-50-4000 ext. 4950 y 555513975203.

* Sin fuente de financiamiento declarada para el desarrollo de la investigación
} 


\section{Abstract}

BGM provides the best fair value estimate, coinciding with the suggestions of other authors. Consequently, it is recommended that Mexican companies know and implement this model, complementing the traditional valuation of discounted cash flows, in order to present relevant information for the decision-making process. JEL Classification: G12, G13, C4, C5, C15

Keywords: Discounted Cash Flows, Real Options as a Geometric Brownian Motion, Real Options as an Arithmetic Brownian Motion, GARCH (1,1), Winters' Method

\section{Introducción}

A partir de la aplicación de la Norma de Información Financiera (NIF) A-6 "Reconocimiento y Valuación" emitida por el Consejo Mexicano de Normas de Información Financieras (CINIF) así como de la Norma Internacional de Información Financiera (NIIF) 16 (conocida por sus siglas en inglés como IFRS, International Financial Reporting Standards) "Medición del Valor Razonable" ha sido imperante para las entidades cuantificar contablemente sus transacciones de la forma más certera para la adecuada toma de decisiones. Es por dicha razón que bajo ambas normatividades se deben reconocer las operaciones al denominado Valor Razonable; el cual se define como: "el monto de efectivo o equivalentes que participantes en el mercado estarían dispuestos a intercambiar para la compra o venta de un activo, o para asumir o liquidar un pasivo, en una operación entre partes interesadas, dispuestas o informadas, en un mercado de libre competencia. Cuando no se tenga un valor de intercambio accesible de la operación debe realizarse una estimación del mismo mediante técnicas de valuación". Cabe resaltar que la regulación es muy ambigua al permitir utilizar cualquier modelo reconocido en el ámbito financiero para determinar el Valor Razonable.

Es por ello que resulta extremadamente importante utilizar el modelo que brinde la mejor aproximación posible del verdadero valor de las partidas que acorde con la normatividad contable, al llevar a cabo una valuación posterior. Especialmente en la actualidad, ya que la mayor parte de las mismas deben presentarse a Valor Razonable, como son los casos de los activos de larga duración, los activos intangibles y el crédito mercantil, el valor de las inversiones en acciones, entre otras.

El modelo aplicado con mayor frecuencia es el de Flujos de Efectivo Descontados, por ser el más conocido, así como por su relativa sencillez en su cálculo; sin embargo, como lo menciona Trigeorgis (1996) las Opciones Reales representan una extensión de la teoría de las Opciones Financieras aplicada sobre activos no financieros.

Esta teoría se basa en la flexibilidad que tiene la administración de cambiar sus decisiones. "En ausencia de esta flexibilidad la distribución de probabilidad del Valor Presente Neto podría ser razonablemente simétrica". "Esta asimetría introducida por la flexibilidad de la administración se le denomina el criterio del Valor Presente Neto expandido, el cual refleja dos componentes: el tradicional o VPN estático proveniente directamente de los flujos de efectivo y la parte activa, la que incorpora el efecto de la competencia, la sinergia que trae un proyecto, la interacción entre diferentes etapas dentro de un proyecto y la respuesta de la administración a todos estos factores. Esta flexibilidad puede ser capturada de una mejor manera en lo que Trigeorgis (1996) denomina como el Valor Presente Neto Expandido:

A pesar de que esta herramienta tiene más de 20 años de haber sido propuesta, en México es muy poco utilizada, no obstante, de que, en múltiples investigaciones, así como en diversas industrias, sobre todo Estados Unidos es utilizada; ya que se ha demostrado que resulta más eficaz que el modelo tradicional de Flujos de Efectivo Descontados.

En la implantación de las Opciones Reales, tanto de forma científica como empírica se asume que el flujo de efectivo se comporta como un Modelo Browniano Geométrico; sin embargo, como lo afirman Lambert y 
Moreno (2017) al igual que Copeland y Antikarov (2001) los flujos de efectivo de una empresa o proyecto y por consecuencia el Valor Presente Neto resultante, pueden ser negativos, por lo que al modelar la trayectoria de estos flujos como un Movimiento Browniano Geométrico, éste se mantendrá negativo o positivo a lo largo de todo el periodo de la valuación, acorde con el valor inicial. No obstante, Alexander, Mo y Stent (2012) afirman que el valor intrínseco de un proyecto no permanecerá positivo necesariamente durante toda su vida, por lo cual sugieren aplicar el modelo de Opciones Reales bajo un Modelo Browniano Aritmético, al utilizar este proceso estocástico, sin importar que se comience con un flujo positivo, el valor del proyecto o de la empresa puede tornarse negativo.

Derivado de lo anterior, el objetivo de la investigación es determinar si el modelo de Opciones Reales bajo dicho proceso aproxima en mayor medida el Valor Razonable, así como los valores de mercado de la empresa, de mercado del capital accionario y consecuentemente, el precio de la acción, en contraste con los modelos de Flujos de Efectivo tradicional y el de Opciones Reales pero acorde con el Modelo Browniano Geométrico.

Este trabajo se compone de las siguientes secciones: primero se efectuará una revisión de las investigaciones previas; en la segunda se describirá el marco teórico de las Opciones Reales bajo el Movimiento Browniano Geométrico y el Movimiento Browniano Aritmético, en el tercer apartado se explica la metodología, en el cuarto se muestran los resultados derivados del análisis, en el quinto las conclusiones y recomendaciones, finalizando el documento con las referencias bibliográficas.

\section{Marco Teórico}

\subsection{Opciones Reales}

Las Opciones Reales representan una extensión de la teoría de las Opciones Financieras aplicada sobre activos no financieros. Como lo explica Trigeorgis (1996), se puede utilizar la metodología de las Opciones Financieras en la valuación de una firma. Al resultado se le denomina el criterio del Valor Presente Neto expandido, el cual refleja dos componentes: el tradicional o VPN estático proveniente directamente de los flujos de efectivo y la parte activa, la que incorpora el efecto de la competencia, la sinergia que resulta de un proyecto, la interacción entre diferentes etapas dentro del proyecto y la respuesta de la administración a todos estos factores. En el presente análisis se aplicó únicamente el modelo a tiempo discreto, es decir el binomial, el cual es utilizado para replicar el comportamiento de un activo financiero. Asimismo, únicamente se evaluaron las opciones de expansión y contracción tanto para el MBG como para el MBA.

\section{Modelos Browniano Geométrico y Browniano Aritmético}

\subsubsection{Modelo Browniano Geométrico}

En cada periodo existen dos posibilidades, el valor del activo puede subir en un factor $\mathrm{u},(u>1)$, o bien bajar en un factor $\mathrm{d},(d<1)$. El modelo binomial se basa en un proceso Bernoulli; acorde con el cual, los choques al alza y a la baja se determinan de la siguiente manera:

$$
\begin{gathered}
u=e^{\sigma * \sqrt{\Delta t}} \\
u=e^{-\sigma * \sqrt{\Delta t}}
\end{gathered}
$$

Dónde:

u es el choque al alza que seguirá el subyacente.

d es el choque a la baja que seguiría el subyacente. 
$\sigma$ es la volatilidad (desviación estándar) de la serie de tiempo del subyacente, la cual se mantiene fija a lo largo del periodo de la valuación.

Para obtener los choques al alza y a la baja, se calculó su volatilidad con el modelo GARCH $(1,1)$ (Autoregressive Conditional Heteroskedasticity), partiendo como punto inicial, el valor obtenido con el modelo de Flujos de Efectivo Descontados, acorde con esa premisa se elaboró el árbol de las Opciones Reales, asumiendo que el proceso estocástico se comporta como una submartingala, dado que se valuó como una opción americana.

En la siguiente sección se expondrá brevemente cómo se determinó la volatilidad para el modelado de las Opciones Reales bajo el proceso en comento; mientras que en la 2.1.2 se explicará el modelo en tiempo discreto, para valuar una opción, dicho modelo fue aplicado para estimar el valor de mercado del capital accionario, tanto bajo el proceso Browniano Geométrico como el Browniano Aritmético. Este último con la solución propuesta por Alexander et al. (2012), el cual será explicado más adelante.

\subsubsection{Cálculo de la volatilidad modelo Browniano Geométrico}

Para estimar la volatilidad histórica de una serie de tiempo, se toma un intervalo fijo (ej. diaria, semanal, mensual, etc.).

Sea:

n es el número de observaciones.

$S_{i}$ el valor del instrumento al final del $i_{t h}$ intervalo.

$\tau$ la longitud del intervalo en años.

El rendimiento correspondiente a un periodo de $i=1,2, \ldots n$, de un instrumento se calcularía de la siguiente forma:

$$
u_{i}=\ln \left(\frac{S_{i}}{S_{i-1}}\right) \text { para } i=1,2, \ldots . n
$$

Ya que $S_{i}=S_{i-1} e^{u_{i}}$, es el valor del subyacente incrementado de forma continua (no anualizado) en el $i_{t h}$ intervalo. El estimador utilizado generalmente para obtener la desviación estándar de $u_{i}$ es:

$$
s=\sqrt{\frac{1}{n-1} \sum_{i=1}^{n}\left(u_{i}-\bar{u}\right)^{2}}
$$

o bien,

$$
s=\sqrt{\frac{1}{n-1} \sum_{i=1}^{n} u_{i}^{2}-\frac{1}{n(n-1)}\left(\sum_{i=1}^{n} u_{i}\right)^{2}}
$$

Dónde:

$\bar{u}$ es la media de las $u_{i} / s$

$n$ es el número de las observaciones.

La desviación estándar del cambio proporcional del precio de un instrumento en un pequeño intervalo de tiempo $\Delta t$ es $\sigma \Delta t$, lo cual es una aproximación de la desviación estándar del cambio proporcional en el precio del instrumento sobre un largo periodo de tiempo T: $\sigma \sqrt{T}$.

Dado que s es un estimador de $\sigma \sqrt{T}, \sigma$ puede ser aproximada como $\sigma^{*}$ de la siguiente forma:

$$
\sigma^{*}=\frac{s}{\sqrt{\tau}}
$$

Cabe señalar que la s no cambia a través del tiempo, y los datos muy antiguos no resultan muy relevantes para predecir el futuro. Es por ello que resulta imperante utilizar la volatilidad dinámica o bien modelos más 
sofisticados como son el Autorregresivo de Heteroscedasticidad Condicional, conocido por sus siglas en inglés como ARCH o el modelo Generalizado Autorregresivo de Heteroscedasticidad Condicional, conocido por sus siglas en inglés como GARCH.

Bollerslev (1986) extendió el trabajo de Engel desarrollando una técnica que le permite a la varianza condicional ser un proceso ARMA, siendo el proceso del error el siguiente:

$$
\varepsilon_{t}=v_{t} \sqrt{h_{t}}
$$

Dónde:

$$
\sigma_{v}^{2}=1 \quad y \quad h_{t}=\alpha_{0}+\sum_{i=1}^{q} \alpha_{i} \varepsilon_{t-1}^{2}+\sum_{i=1}^{p} \beta_{i} h_{t-i}
$$

Ya que $v_{t}$ es un proceso de ruido blanco, independiente de las realizaciones pasadas por lo tanto su media condicional e incondicional es igual a 0 .

El modelo general ARCH(p,q) es llamado Generalized Autoregressive Conditional Heteroscedastic GARCH(p,q), e incorpora tanto los componentes autorregresivos como de medias móviles en la varianza heteroscedástica.

Es condición que todos los coeficientes de la ecuación anterior deben ser positivos, y para asegurar que la varianza sea finita, las raíces características deben encontrarse dentro del círculo unitario.

El factor clave de este modelo es que la varianza condicional de las perturbaciones de la secuencia de $\left\{y_{t}\right\}$ constituyan un proceso ARMA (Autorregresivo y de Promedios Móviles).

El modelo $\operatorname{GARCH}(1,1)$ fue propuesto por Bollerslev en 1986. Este calcula $\sigma_{n}^{2}$ a partir del promedio de la varianza de largo plazo $\mathrm{V}$, así como de $\sigma_{n-1}^{2}$ y de $u_{n-1}$. La ecuación que le representa es la siguiente:

$$
\sigma_{n}^{2}=\gamma V+\alpha u_{n-1}^{2}+\beta \sigma_{n-1}^{2}
$$

Donde es la ponderación que se le da a $\mathrm{V}, \alpha$ es la ponderación otorgada a $u_{n-1}^{2}, \mathrm{y} \beta$ es la ponderación aplicada sobre $\sigma_{n-1}^{2}$, las ponderaciones deben sumar 1 .

Estableciendo $\omega$ como $\gamma \mathrm{V}$, este modelo puede ser escrito de la siguiente manera:

$$
\sigma_{n}^{2}=\omega+\alpha u_{n-1}^{2}+\beta \sigma_{n-1}^{2}
$$

Una vez estimados los coeficientes de la ecuación, es posible obtener la varianza de largo plazo dividiendo: $\omega(1-\alpha-\beta)$. Para la estimación de estos parámetros se puede aplicar el método de máxima verosimilitud (maximum likelihood), en el cual se obtienen los valores de los parámetros de forma que se maximice la probabilidad de ocurrencia de los datos.

\subsubsection{Valuación de Opciones en Tiempo Discreto, Modelo Binomial}

Una técnica muy utilizada para describir el proceso que sigue una opción sobre un activo subyacente es el modelo binomial, el cual consiste en construir un árbol binomial, con el que se muestra las diferentes trayectorias que puede seguir el subyacente durante la vida de la opción. Esta propuesta es similar a la realizada por John Carrington Cox, Stephen Ross y Mark Edward Rubinstein en 1979. El valor de la opción puede obtenerse a través del modelo binomial o calculando el portafolio de réplica.

Un argumento similar puede ser utilizado para las opciones, pero es necesario suponer que no existen oportunidades de arbitraje, "No existen oportunidades de arbitraje si y sólo si existe una medida de probabilidad neutral al riesgo"” (Pliska, 1998).

\footnotetext{
${ }^{2}$ Es una probabilidad sintética, bajo la cual el valor esperado del instrumento es el justo ó el de mercado.
} 
Para soportar lo anterior, también es importante suponer que los individuos son neutrales al riesgo ${ }^{3}$, por lo que no es indispensable que una inversión ofrezca una prima adicional por el riesgo tomado, en consecuencia, la tasa mínima de retorno que se espera sobre una inversión es la tasa libre de riesgo y el valor futuro de las opciones deberá ser descontado a esta tasa.

Pero tanto el mercado como los compradores deben evitar que sea un medio para obtener ganancias extranormales, para lo cual se deben eliminar todas las posibles oportunidades de arbitraje.

Por dicha razón, el valor de este instrumento debe ser el "justo", es decir, el que sea percibido todo el mercado. Para conocer ese valor deben aislarse todos los posibles factores de subjetividad, como es el caso de la tasa de descuento utilizada de acuerdo con el grado de aversión al riesgo de cada inversionista; el modelo que toma en cuenta este problema es el de la valuación por "martingala", el cual descuenta los valores esperados a la tasa libre de riesgo y bajo unas probabilidades sintéticas "probabilidad neutral al riesgo" (q), las cuales se encuentran determinadas por $\mathrm{u}$, d y la tasa libre de riesgo (r).

$$
q=\frac{e^{r * t}-d}{u-d}
$$

La incorporación de la volatilidad en la fórmula de la probabilidad neutral al riesgo, mediante los parámetros u y d, los cuales se calculan como se mostró anteriormente.

Una vez calculados dichos parámetros, puede determinarse la prima de la opción. El primer paso consiste en modelar la trayectoria que seguirá el valor del subyacente durante la vigencia de la opción. Este valor puede aumentar en u o bajar a d.

Posteriormente se elabora el árbol de la opción, comenzando con los últimos nodos del árbol. Se determinará para cada uno de los nodos finales el valor intrínseco de la opción (el máximo entre la diferencia del subyacente y el precio de ejercicio o cero). Cabe señalar que la metodología seguida para determinar el valor de las opciones fue aplicada al ser modeladas como un Movimiento Browniano Geométrico así como un Movimiento Browniano Aritmético.

En una opción americana, el poseedor tiene el derecho de ejercer la opción en cualquier momento $\tau$, si se realiza el contrato en el tiempo t, y la madurez es en el tiempo $\mathrm{T}$, el ejercicio puede efectuarse en el lapso $t \leq \tau \leq T$. Un proceso de este tipo se diferencia de la martingala en que el valor esperado puede ser mayor o igual que el valor actual.

La mecánica para determinar el valor de una opción americana es similar al de la europea, varía únicamente en el cálculo de los nodos intermedios, ya que por las características de estas opciones el poseedor de la posición larga puede ejercerla en cualquier momento hasta su vencimiento, por lo anterior puede escoger el máximo entre el valor intrínseco de la opción y el valor descontado bajo la probabilidad neutral al riesgo.

\subsubsection{Modelo Browniano Aritmético}

Como se comentó con anterioridad, un supuesto estándar cuando se valúan las Opciones Financieras en mercados de activos negociables es que el precio del subyacente se comporta como un Movimiento Browniano Geométrico.

No obstante, como lo mencionan Copeland y Antikarov (2001, Capítulo 5) el valor de los flujos de efectivo de una empresa o proyecto pueden ser negativos, lo cual por consecuencia tiene una implicación de gran relevancia para las Opciones Reales. Derivado de lo anterior proponen modelarlo como un proceso aditivo, es decir como un Movimiento Browniano Aritmético, bajo el cual, aunque el valor inicial sea positivo, se puede

\footnotetext{
${ }^{3}$ Un individuo es neutral al riesgo, cuando no espera recibir una prima adicional sobre su inversión en un activo riesgoso.

${ }^{4} \mathrm{El}$ que se valúe por martingala, implica que el valor de una variable aleatoria $\mathrm{Z}$ en el tiempo $\mathrm{s}+\mathrm{t}$ de acuerdo con la información disponible es igual al valor que tenía la variable aleatoria en el tiempo t.
} 
tornar en algún momento de la trayectoria de la valuación negativo. Alexander, Mo y Stent (2012) efectúan la derivación partiendo del supuesto de neutralidad al riesgo, y por consecuencia del descuento de los flujos del árbol de las opciones bajo una probabilidad neutral al riesgo, así como el uso de la tasa libre de riesgo para descontar dichos flujos.

Considerando que el subyacente se comporta como un Movimiento Browniano Aritmético (MBA), el cambio en el valor inicial del proyecto $V_{0}$ en los periodos posteriores $V_{t}$ estaría dado por la siguiente ecuación estocástica:

$$
d V_{t}=\alpha d_{t}+\beta d Z_{t}
$$

Con $\alpha$ y $\beta>0$ constantes y siendo $Z_{t}$ un proceso browniano estándar con medida de probabilidad objetiva p.

El MBA es apropiado cuando los cambios en el valor de un proyecto pueden ser representados como la suma de una tendencia lineal y ruidos blancos aditivos, asimismo el pronóstico de los valores futuros en el tiempo puede ser modelado como una distribución de probabilidad normal. La distribución normal permite valores negativos de los proyectos.

De igual forma proponen que los flujos de efectivo libres sean formulados inicialmente como un rendimiento por dividendos (dividend yield) pagando una tasa constante $\delta \geq 0$, calculados sobre el importe de valor del proyecto o de los flujos de efectivo $V_{t}$. En forma diferencial el proceso del dividendo se representaría de la siguiente manera:

$$
d D_{t}=\delta V_{t} d t
$$

De conformidad con la fórmula de Black y Scholes, un bono con un rendimiento constante, compuesto continuamente a la tasa nominal $r>0$ por unidad de tiempo, el cual es aplicado en un mercado secundario normalizado. El proceso de ganancias descontadas en la ecuación diferencial se representa de la siguiente manera.

$$
d \check{G}_{t}=d\left(e^{-r t} V_{t}\right)+e^{-r t} d D_{t}
$$

Como se puede ver en la ecuación anterior, el cambio diferencial en la ganancia es igual a la variación en el tiempo en el valor de los flujos a valor presente más los cambios diferenciales en los dividendos.

$\mathrm{Al}$ aplicar la regla del producto y sustituyendo en el proceso del valor (ecuación (12)) y el proceso del dividendo (ecuación (13)), se obtiene la siguiente ecuación diferencial.

$$
d \check{G}_{t}=-r e^{-r t} V_{t} d t+e^{-r t} d V_{t}+e^{-r t} \delta V_{t} d t=e^{-r t} \beta\left(\theta_{t} d_{t}+d Z_{t}^{*}\right)
$$

Dónde:

$$
\begin{gathered}
\theta_{t}=\left[\alpha-(r-\delta) V_{t}\right] / \beta \\
d Z_{t}^{*}=\theta_{t} d t+d Z_{t}
\end{gathered}
$$

Cabe recordar que $\alpha$ y $\beta$ son constantes $>0$ definidas en la ecuación diferencial estocástica del valor del proyecto (Ecuación (12)).

Acorde a lo establecido por Alexander et al. (2012) por el teorema de Girsanov ${ }^{5}$ se puede demostrar la existencia de una medida de probabilidad neutral al riesgo, q, para cualquier nuevo proceso $d Z_{t}^{*}$, siendo éste un movimiento Browniano estándar.

\footnotetext{
${ }^{5}$ Este teorema describe cómo la dinámica de los procesos estocásticos cambia cuando la medida original se modifica por una medida de probabilidad equivalente. Particularmente en la teoría de opciones, explica cómo se efectúa la conversión de la medida física, es decir la probabilidad de que el precio del subyacente cambie hacia una medida de probabilidad neutral al riesgo.
} 
Adicionalmente dichos autores establecen que con el propósito de derivar las fórmulas analíticas de un Call y Put Europeos, así como la distribución de $V_{t}$ bajo una medida de probabilidad neutral al riesgo q, resulta necesario reajustar la ecuación (15) de la siguiente forma:

$$
\beta d Z_{t}^{*}=-(r-\delta) V_{t} d t+d V_{t}
$$

Esta es una ecuación de Langevin y tienen solución (Karatzas y Shreve (1991)).

$$
V_{t}=e^{(r-\delta)} V_{0}+\beta e^{(r-\delta) t} \int_{0}^{t} e^{-(r-\delta) s} d Z_{t}^{*}
$$

En el tiempo t acorde a una medida de probabilidad neutral al riesgo q, $V_{t}$ es una variable aleatoria normal con media $\mu_{t}$ y varianza $\sum_{t}^{2}$, aplicando la isometría de Ito (Óksendal, (1998)).

$$
\begin{gathered}
\mu_{t}=e^{(r-\delta)} V_{0}(47) \text { Tasa del incremento en el valor del proyecto promedio. } \\
\qquad \begin{array}{c}
\sum_{t}^{2}=\frac{\beta^{2}}{2(r-\delta)}\left(e^{2(r-\delta)} t-1\right) \text { si } r \neq \delta \text { volatilidad del proyecto } \\
=\beta^{2 t} \text { si } r=\delta
\end{array}
\end{gathered}
$$

\subsubsection{Modelado de las salidas de efectivo con una función ajustada}

Considerando que un Call o Put europeo con madurez t unidades de tiempo, con precio de ejercicio igual a $\mathrm{K}$ tiene un valor contingente dado por la expresión $\max \left(V_{t}-K, 0\right)$ para los Call o el $\max \left(K-V_{t}, 0\right)$ para los Put. Así como salidas constantes de efectivo proporcionales al valor de un proyecto $V_{t}$ debido a los pagos de dividendos, Alexander et al. (2012) modelaron la opción como un movimiento Browniano y a las salidas de flujo de efectivo se les incorporó cierta flexibilidad mediante una función ajustada, de la manera en que se muestra a continuación.

$$
V_{t}=g(t) V_{t}+(1-g(t)) V_{t}
$$

La función $g(t)$ es escalonada determinística, la cual muestra el valor remanente del proyecto después de la salida de los flujos de efectivo. El complemento $(1-g(t))$ es la proporción que ha sido pagada en efectivo en el tiempo t. Mientras que el valor remanente del proyecto es denotado como $V_{t}^{g}$. Dicho valor remanente del proyecto sería de la siguiente forma:

$$
V_{t}^{g}=g(t) V_{t}
$$

La función $g(t)$ es establecida como no negativa y no creciente en el tiempo; con $g(0)=1$. En el caso de que el proyecto o empresa tenga una vida finita $T$ sin valor terminal, la función $g(T)=0$. Asimismo, dicha función posee brincos en tiempo discreto, los cuales se encuentran pre-determinados.

\subsubsection{Fórmulas de los Calls y Puts sobre activos escalonados}

Un Call y Put europeo sobre un activo escalonado, con vencimiento en el tiempo $\tau$ y precio de ejercicio $\mathrm{K}$, tiene un valor intrínseco igual al máx $\left(V_{\tau}^{g}-K, 0\right)$ para un Call o máx $\left(K-V_{\tau}^{g}, 0\right)$ para el Put. Sus valores respectivamente, descontados a la tasa libre de riesgo, incorporando la proporción que representa la salida de efectivo y bajo la probabilidad neutral al riesgo serían de la siguiente forma:

$$
\begin{gathered}
c_{M V A}^{g}=e^{-r t} E^{q}\left[\max \left(V_{\tau}^{g}-K, 0\right)\right]=e^{-r t} g(\tau) E^{q} \max \left[V_{\tau}^{g}-K / g(\tau), 0\right] \\
p_{M V A}^{g}=e^{-r t} E^{q}\left[\max \left(K-V_{\tau}^{g}, 0\right)\right]=e^{-r t} g(\tau) E^{q} \max \left[\frac{K}{g(\tau)}-V_{\tau}^{g}, 0\right]
\end{gathered}
$$




\subsubsection{Desarrollo del modelo en tiempo discreto}

En este trabajo se aplicó el análisis en tiempo discreto, por lo cual las opciones se valuaron como americanas. Al utilizar el modelo binomial se facilita el modelado, ya que pueden evaluarse como Europeas y/o Americanas.

Las salidas de efectivo modeladas dentro del árbol binomial se realizan de una forma escalonada, la nueva escala de tiempo se encuentra definida por una función estrictamente creciente b:

$$
s=b(t)=\int_{0}^{t} e^{-2(r-\delta)} d s=-\frac{1}{2(r-\delta)}\left(1-e^{-2(r-\delta) t}\right) \text { tal que } t \epsilon[0, \tau]
$$

El nuevo rango del lapso de tiempo escalonado es: $s \epsilon[b(0, b(\tau))]=[0, b(\tau)]$. Lo cual, acorde con Alexander et al. (2012) estos puntos serán necesarios para invertir la escala de tiempo original, para ello la función inversa de $b$ es requerida, a la cual se le denotará como:

$$
t=a(s)=b^{-1}(s)=-\frac{1}{2(r-\delta)} \log _{e}(1-2(r-\delta) s) \quad \text { tal que } s \in[0, b(\tau)]
$$

La aproximación del tiempo para el modelo binomial se puede definir como un movimiento Browniano en el intervalo $[0, b(\tau)]$ en $\mathrm{N}$ intervalos de longitud constante $k=b(t) / N$, generando puntos de tiempo $n k$ para $n=0, \ldots N$. En cuyo caso el choque al alza se obtendría de la siguiente forma: $u=\sqrt{k}$, acorde con el cual son igualmente probables los choques al alza como a la baja por unidad de tiempo k. A continuación, se presentan las imágenes de los árboles binomiales bajo un Movimiento Browniano Aritmético. La relación entre el lapso de tiempo entre los nodos es mostrada en la Imagen 1, esto con el objeto de completar la construcción y obtener el árbol binomial que simule la trayectoria representada en la ecuación (23). Para aproximar todos los nodos mostrados en uno de los periodos de tiempo de la ecuación estocástica (24) la $\mathrm{n}$ es multiplicada por $e^{(r-\delta) \alpha(n k)}$ (Imagen 2).

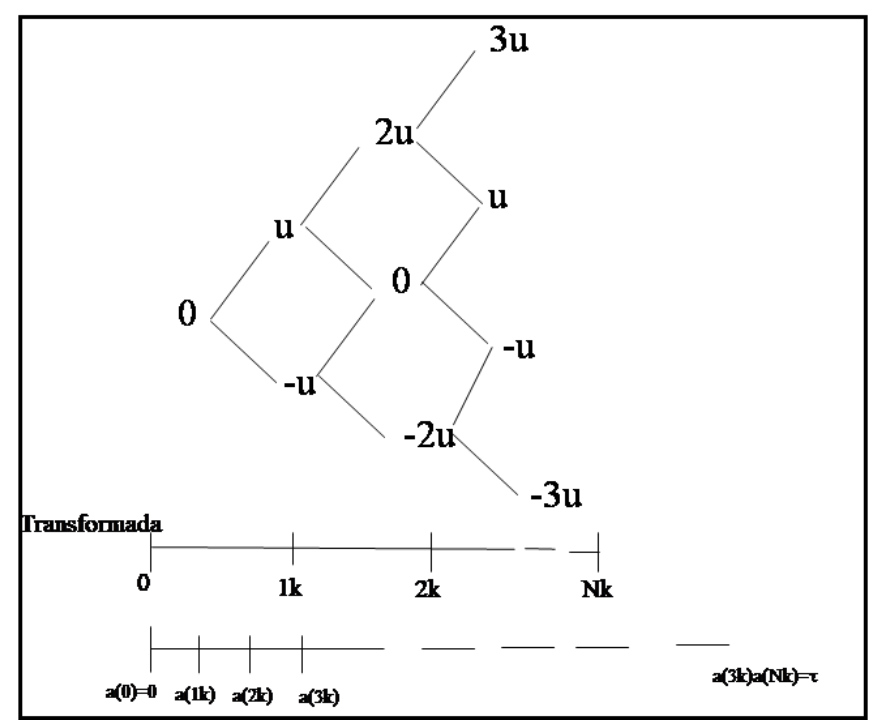

Imagen 1. Recombinación del árbol binomial para una proceso browniano estándar de conformidad con la ecuación (23)

Fuente: Alexander et al. (2012) 


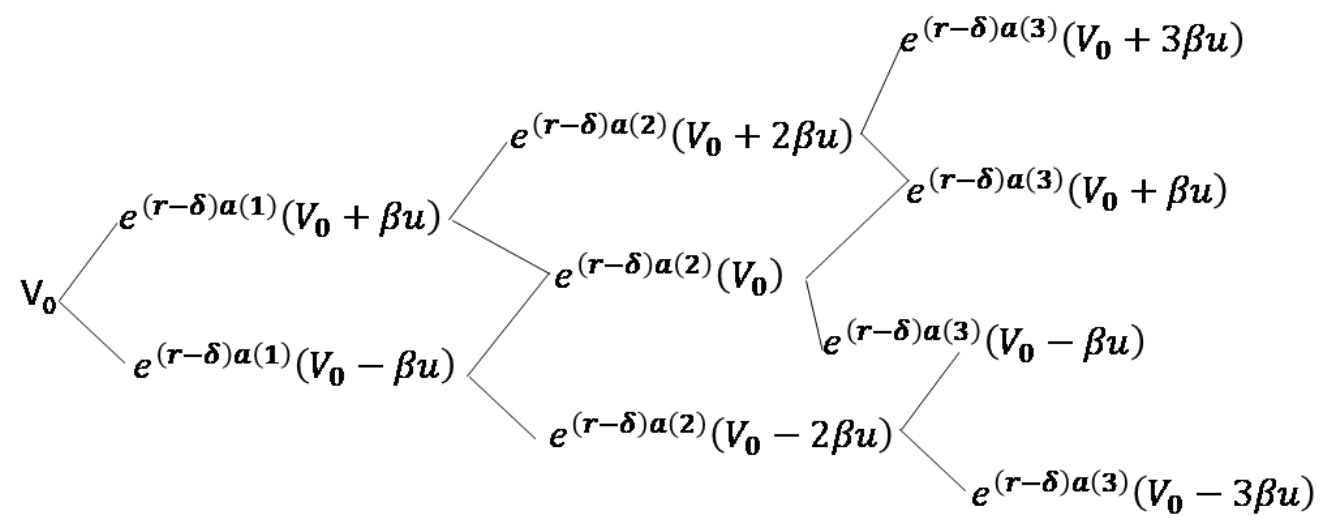

Imagen 2. Recombinación del árbol acorde con la ecuación (24)

Fuente: Alexander et al. (2012)

A continuación, se describe la metodología seguida, así como los resultados de la aplicación empírica de los modelos explicados en esta sección, pero considerando la información de empresas mexicanas que cotizan en la Bolsa Mexicana de Valores.

\section{Metodología y Resultados}

El objetivo de este estudio es encontrar el modelo que brinde la mejor estimación asequible del Valor Razonable de las partidas que componen los estados financieros; particularmente resulta sumamente importante que la administración de la empresa concientice que debe determinar el valor posterior de las transacciones considerando la ambigüedad contenida en la normatividad contable para su cálculo.

Por lo cual, el propósito es analizar tres metodologías comparando los resultados al calcular el valor de mercado de la empresa, el valor de mercado del capital accionario y el precio de la acción, comparando esta última variable con el último precio cotizado.

Para cumplir con dicho objetivo se analizó la información financiera (estado de situación financiera, estado de resultados y flujos de efectivo) de 101 entidades no financieras que cotizan en la Bolsa Mexicana de Valores que han cotizado durante el periodo de febrero a marzo de 2019); no obstante, se tuvieron que descartar 61 compañías, ya que no poseían la cantidad de información necesaria para realizar las valoraciones. Para las proyecciones y cálculos se tomó la información trimestral por el periodo comprendido del primer trimestre de 2011 al cuarto trimestre de 2018.

Las empresas que cumplieron con la cantidad de información requerida para aplicar los modelos descritos fueron 40, las cuales se enuncian a continuación (Tabla 1):

Tabla 1. Empresas utilizadas como muestra.

\begin{tabular}{|c|c|c|c|c|}
\hline \multicolumn{5}{|c|}{ Nombre de las empresas } \\
\hline ALSEA & CARSO & HERDEZ & ROTOPLAS & BIMBO \\
\hline AMÉRICA MÓVIL & CEMEX & HOMEX & URBI & GIGANTE \\
\hline ARA & CHEDRAUI & HOTELES EXPRESS & VITRO & MINERA FRISCO \\
\hline ARISTOS & CIE & INTERCERAMIC & WALMART & MOCTEZUMA \\
\hline AUTLAN & COCA COLA FEMSA & KIMBERLY & FEMSA & BENAVIDES \\
\hline AXTEL & COLLADO & KUO & FRAGUA & FAMSA \\
\hline AZTECA & CRM & LALA & GENOMMA LAB & MÉDICA SUR \\
\hline BACHOCO & ELEKTRA & LIVERPOOL & CABLEVISIÓN & GRUPO MÉXICO \\
\hline \multicolumn{2}{|c}{ Fuente: Elaboración propia. } \\
\end{tabular}




\subsection{Valuación Tradicional de Flujos de Efectivo Descontados}

En cualquier economía en la que los individuos, empresas y gobiernos tengan presente la oportunidad del tiempo, el valor del dinero será un concepto importante, ya que las preferencias de consumo de los individuos premian a los que sacrifican el consumo actual por el consumo futuro.

El valor del dinero no está dado solamente por el monto del mismo, sino también por el momento en el que se recibe o se gasta, de tal manera que la programación en el tiempo de los flujos de efectivo que se esperan recibir o desembolsar determinará la decisión de los individuos de consumir o invertir en el presente.

\subsubsection{Valor Presente (VP)}

El valor presente de un importe a pagar en períodos futuros, es la cantidad que si se tuviera disponible hoy, crecería hasta igualar la suma futura. Esto significa que éste se calcula mediante un proceso de descuento de los flujos a recibir en un futuro a la tasa de interés ofrecida por alternativas comparables, según se muestra a continuación:

$$
V P=\frac{V F}{(1+i)}
$$

Dónde

VP es el Valor Presente.

VF es el Valor Futuro.

i es la tasa de descuento, representa el costo de oportunidad de la inversión.

Si los flujos futuros comprenden varios períodos entonces:

$$
V P=\frac{V F}{(1+i)^{n}}
$$

Dónde:

n número de períodos.

El concepto de valor presente es muy importante ya que en las decisiones de inversión, financiamiento y política de dividendos necesariamente se ven involucrados flujos de dinero a recibir o pagar en un futuro o ambos.

El valor presente de una serie de flujos es sino la suma de los valores futuros de cada uno de los pagos, según se muestra a continuación:

$$
V P=\sum_{t=0}^{t=n} \frac{V F_{t}}{(1+i)^{t}}
$$

Si los flujos de efectivo a recibir o a pagar se realizan durante un número infinito de períodos entonces el término usado es la perpetuidad y la fórmula para el cálculo de su valor presente es simplemente el valor de la cantidad recibida cada período (A) entre la correspondiente tasa de interés (i):

$$
V P=\frac{A}{i}
$$

Es importante mencionar que la fórmula de perpetuidad calcula el VP del total de flujos un periodo atrás del momento en que se comienzan a recibir o a pagar estos flujos. Por ejemplo, si se tiene que pagar una cantidad constante a perpetuidad a partir del próximo año y se aplica la fórmula anterior para obtener el valor presente de los flujos, el resultado estaría a pesos de hoy, pero si el flujo se comienza a pagar a partir del segundo año, entonces el valor presente de los flujos a perpetuidad estará a pesos del próximo año.

Una vez que se explicaron los elementos importantes en el cálculo del valor del dinero en el tiempo se 
hará una breve descripción del modelo de Flujo de Efectivo Libre Descontado (FED) como herramienta de valuación.

\subsubsection{Flujo de Efectivo Libre Descontado (FED)}

Existen diversas formas de aplicar el modelo de FED; sin embargo, el enfoque empresarial es el más utilizado en la práctica. Esta perspectiva valúa el capital de la compañía como el valor de las operaciones de la empresa menos el valor de la deuda y otras obligaciones con mayor preferencia al capital común (como acciones preferentes). Puede aplicarse a diferentes niveles, es decir puede emplearse en toda la compañía o sobre unidades individuales y es consistente con el proceso de presupuesto de capital de la mayoría de las compañías. A este respecto, para poder calcular el FED, se debe obtener el Flujo de Efectivo Libre (FEL), mismo que resulta del siguiente proceso: restar a la utilidad operativa los impuestos operativos, posteriormente sumar a la utilidad operativa después de impuestos las partidas virtuales, obteniendo así el flujo bruto. A este flujo se le suman o restan (según sea el caso) los cambios en el capital de trabajo neto ${ }^{6}$ y finalmente se suman/restan los cambios en la propiedad, planta y equipo.

Por lo tanto, el FEL es el flujo disponible que tiene la empresa para cubrir los costos de sus fuentes de financiamiento y/o amortizar deuda y/o repartir dividendos.

Este flujo de efectivo se calculó para cada una de las 40 entidades seleccionadas, proyectando cada uno de sus elementos anualmente por el lapso de tiempo de 2019 a 2028. En primera instancia se aplicó el modelo de Flujos de Efectivo Descontados, para ellos, los ingresos se estimaron con el modelo de Winters-Multiplicativo. Posteriormente se obtuvieron los costos y gastos operativos como una proporción histórica de los ingresos, la cual se mantuvo constante a lo largo del periodo de la proyección.

El capital de trabajo neto y los gastos de capital se proyectaron tomando la relación entre: ingresos operativos con cuentas por cobrar (ingresos por cuentas por cobrar), inventarios con el costo de ventas; otros activos corrientes con ingresos y cuentas por pagar con el costo de ventas. Las inversiones en propiedad, planta y equipo para la producción y mejora se determinaron mediante su relación con los ingresos. En consecuencia, se utilizaron las siguientes razones financieras:

$$
\begin{gathered}
\text { Razon de Cuentas por Cobrar = Ingresos/Cuentas por Cobrar } \\
\text { Razon de Inventarios }=\text { Costo de Ventas/Inventarios } \\
\text { Razon de Cuentas por Pagar }=\text { Costo de Ventas/Cuentas por Pagar } \\
\text { Razon de Otros Activos = Ingresos/Otros Activos Corrientes }
\end{gathered}
$$

Para la proyección de las inversiones en propiedad, planta y equipo se utilizó la rotación de propiedad, planta y equipo (la relación entre los ingresos y la propiedad, planta y equipo).

Una vez proyectados los flujos de cada periodo, se tomó el del último año proyectado de forma explícita como una perpetuidad sin crecimiento. Como se mencionó la perpetuidad, trae a valor presente un periodo el importe de los flujos que se generarán a perpetuidad, por lo cual, este valor se descontó posteriormente a pesos de 2019. Asimismo, cada uno de los flujos se descontaron a pesos de 2019, finalmente al sumarse, se obtuvo el valor de mercado de la empresa. A dicho importe se le restó el valor de la deuda con costo reportado en el estado de situación financiera al 31 de diciembre de 2018, obteniendo con ello el valor de mercado del capital accionario.

\footnotetext{
${ }^{6}$ El capital de trabajo neto se calcula sumando a la Cartera de Clientes Neta los Inventarios Netos y restando el saldo de Proveedores y Acreedores.
} 
Por consistencia con la definición de flujos de efectivo, la tasa de descuento aplicada usualmente al FEL debe reflejar el costo de oportunidad de los acreedores y accionistas ponderado por la proporción que su aportación hace al financiamiento de la empresa con respecto al valor total. Esta tasa es denominada por sus siglas en inglés como WACC (Weighted Average Cost of Capital) y se calcula de la siguiente manera:

$$
W A C C=K d^{*} \frac{V D}{V F}+K a \frac{V A}{V F}
$$

Dónde:

$K_{d}^{*}$ representa el costo de la deuda, el cual puede ser medido a través de la obtención de la tasa implícita en los desembolsos futuros provenientes del pago de intereses y capital, menos los beneficios fiscales que proporcionan la parte deducible de los intereses a cargo.

VD valor de mercado de la deuda.

VA Valor del mercado del capital accionario.

VF es la suma de VD y VA es decir el valor de mercado de la empresa.

$E\left(r_{j}\right)$ es la tasa mínima de retorno que esperan los accionistas de acuerdo con el riesgo que representa esta inversión. Este costo puede ser aproximado a través del modelo conocido por sus siglas en inglés como CAPM (Capital Asset Pricing Model), el cual mide la relación riesgo-rendimiento, es decir, la tasa de retorno esperada sobre una acción es igual a la tasa libre de riesgo (el premio por el cambio del consumo de hoy por el consumo de mañana) más una prima por riesgo (es la compensación por el riesgo de tomar la inversión):

$$
E\left(r_{j}\right)=R_{f}+\left[E\left(R_{m}-R_{f}\right)\right] \beta_{j}
$$

Dónde:

$E\left(r_{j}\right)$ representa el rendimiento esperado sobre la inversión $\mathrm{j}$, tal que $j=1, \ldots n$.

$R_{f}$ tasa libre riesgo, se consideró el rendimiento promedio anual ofrecido por la tasa de bonos gubernamentales a 10 años emitidos por el Gobierno Federal de los Estados Unidos Mexicanos, por el periodo comprendido de enero de 2008 a diciembre de 2018.

$R_{m}$ representa el rendimiento esperado de una amplia cartera de acciones en el mercado. En este caso se utilizó el S\&P/BMV IPC sobre el cual se calculó el rendimiento ofrecido por el mismo, utilizando consistentemente la serie de tiempo mensual de enero de 2008 a diciembre de 2018, posteriormente este rendimiento se anualizó de forma simple. Ambos parámetros se obtuvieron de la página de Internet del Banco de México.

Beta es una medida del riesgo que refleja la sensibilidad del precio de las acciones de una empresa ante los movimientos del mercado de valores en su conjunto. En este estudio en particular como todas las entidades que cotizan en la BMV su beta se obtuvo de la base de Bloomberg.

La WACC se determinó tomando los valores de la deuda con costo, así como del capital contable, tomados del estado de situación financiera al 31 de diciembre de 2018. Debido a que no se poseía la información detallada del costo de la deuda, éste se aproximó dividiendo el importe de los gastos por intereses del último ejercicio reportado (2018) entre el saldo del pasivo con costo.

\subsubsection{Método de Winters}

De conformidad con la serie de tiempo de ingresos de cada una de las 40 empresas seleccionadas, se proyectaron por el periodo comprendido del primer trimestre de 2019 al cuarto trimestre de 2028 con el modelo de HoltWinters. Este método estudia la serie temporal, y la divide en tres componentes: lineal, cíclico y de tendencia. Esta metodología disminuye los componentes cíclicos y de tendencia en comento, mediante un índice de tendencia y variables decrecientes. Se aplican cinco ecuaciones, las que se presentan a continuación.

1. Serie exponencial disminuida. 
2. Pronóstico de la tendencia.

3. Pronóstico cíclico.

4. Pronóstico de la estacionalidad.

5. Serie previsión, p, periodos en el futuro.

Se calcularon los componentes aplicando la metodología de máxima verosimilitud, con el fin de minimizar la varianza residual.

A continuación (Tabla 2), se presenta el valor de mercado de cada una de las empresas, calculado con el modelo de Flujos de Efectivo Descontados, así como el costo promedio ponderado de capital (WACC).

Tabla 2. Valor de mercado del capital accionario por el modelo de FED y la WACC correspondiente. (cifras en pesos)

\begin{tabular}{|c|c|c|c|c|c|}
\hline $\begin{array}{c}\text { Nombre de } \\
\text { la empresa }\end{array}$ & $\begin{array}{c}\text { Valor de la } \\
\text { empresa bajo } \\
\text { flujos de efectivo } \\
\text { descontados }\end{array}$ & $\begin{array}{c}\text { Costo } \\
\text { Promedio } \\
\text { Ponderado } \\
\text { de Capital }\end{array}$ & $\begin{array}{c}\text { Nombre de } \\
\text { la empresa }\end{array}$ & $\begin{array}{c}\text { Valor de la } \\
\text { empresa bajo } \\
\text { flujos de efectivo } \\
\text { descontados }\end{array}$ & $\begin{array}{c}\text { Costo } \\
\text { Promedio } \\
\text { Ponderado } \\
\text { de Capital }\end{array}$ \\
\hline ALSEA & $6,474,338,977$ & $5.88 \%$ & GIGANTE & $23,407,826,224$ & $4.92 \%$ \\
\hline AMÉRICA MÓVIL & $60,209,780,164$ & $2.76 \%$ & GRUPO MÉXICO & $25,123,057,033$ & $2.63 \%$ \\
\hline ARA & $5,437,294$ & $3.48 \%$ & HERDEZ & $9,912,914,024$ & $6.17 \%$ \\
\hline ARISTOS & $108,934,656$ & $5.82 \%$ & HOMEX & $-2,836,800$ & $8.82 \%$ \\
\hline AUTLAN & $2,747,934,325$ & $5.82 \%$ & HOTELES EXPRESS & $37,520,392,981$ & $5.65 \%$ \\
\hline AXTEL & $7,507,608,314$ & $6.33 \%$ & INTERCERAMIC & $2,983,160,536$ & $2.58 \%$ \\
\hline AZTECA & $1,782,665,665$ & $6.19 \%$ & KIMBERLY & $115,287,190$ & $1.89 \%$ \\
\hline BACHOCO & $10,075,839,625$ & $7.52 \%$ & KUO & $2,602,152,865$ & $4.43 \%$ \\
\hline BENAVIDES & $153,415,013$ & $9.84 \%$ & LALA & $246,685,678$ & $5.44 \%$ \\
\hline BIMBO & $66,654,110,259$ & $5.84 \%$ & LIVERPOOL & $13,343,886,781$ & $4.01 \%$ \\
\hline CABLEVISIÓN & $188,574,448,415$ & $3.90 \%$ & MÉDICA SUR & $362,120,440$ & $8.19 \%$ \\
\hline CARSO & $49,051,781,090$ & $3.41 \%$ & MINERA FRISCO & $7,963,431,047$ & $1.69 \%$ \\
\hline CEMEX & $16,680,948,043$ & $1.95 \%$ & MOCTEZUMA & $18,609,551,531$ & $1.01 \%$ \\
\hline CHEDRAUI & $33,317,774,459$ & $3.75 \%$ & ROTOPLAS & $9,299,708$ & $4.32 \%$ \\
\hline CIE & $1,031,191,978$ & $0.87 \%$ & URBI & $2,277,107$ & $1.69 \%$ \\
\hline COCA COLA FEMSA & $1,450,217,762,654$ & $3.15 \%$ & VITRO & $19,927,097,054$ & $3.72 \%$ \\
\hline COLLADO & $456,588,239$ & $9.54 \%$ & WALMART & $289,997,522,098$ & $4.68 \%$ \\
\hline CRM & $175,787,788$ & $7.77 \%$ & FEMSA & $27,968,738,244$ & $2.73 \%$ \\
\hline ELEKTRA & $27,373,459,438$ & $3.97 \%$ & FRAGUA & $6,654,350,620$ & $2.13 \%$ \\
\hline FAMSA & $-109,093,087$ & $6.71 \%$ & GENOMMA LAB & $82,081,595$ & $2.42 \%$ \\
\hline
\end{tabular}

Fuente: Elaboración propia

Una vez que se determinó el valor de cada una de las empresas con el modelo de Flujos de Efectivo Descontados, se calculó su volatilidad a través del método de GARCH $(1,1)$, mediante la cual se obtuvieron los choques al alza y a la baja de cada empresa, así como la probabilidad neutral al riesgo (ecuaciones (1), (2) y (11)). Se elaboró el árbol binomial teniendo como valor inicial el proveniente de descontar los flujos de efectivo libres con la WACC, pero en este caso, el modelado se realizó siguiendo la trayectoria de un Movimiento Browniano Geométrico y posteriormente se determinó el árbol de las Opciones Reales considerando únicamente las opciones de expansión y contracción. El incremento/decremento porcentual en el flujo de efectivo de cada uno de los nodos se obtuvo calculando el coeficiente de correlación entre las inversiones de capital y el flujo de efectivo operativo, por los trimestres correspondientes de 2009 a 2018, estos porcentajes se obtuvieron para cada una de las compañías.

Resulta muy importante señalar que para descontar los flujos de las opciones, de conformidad con lo establecido por Trigeorgis (1996) y Alexander et al (2011) se aplica la probabilidad neutral al riesgo y la tasa 
libre de riesgo, es este caso se utilizó el rendimiento que brindan los bonos a 10 años emitidos por el Gobierno Federal Mexicano en febrero de 2019, 8.49

En la Tabla 3 se muestra el valor de mercado del capital accionario mediante las Opciones Reales como un proceso Browniano Geométrico, así como la volatilidad calculada con la ecuación (10).

Tabla 3. Valor de mercado del capital accionario calculado con el MBG y la Volatilidad de los ingresos modelo de GARCH $(1,1)$. (cifras en pesos)

\begin{tabular}{|c|c|c|c|c|c|}
\hline $\begin{array}{l}\text { Nombre de } \\
\text { la empresa }\end{array}$ & $\begin{array}{c}\text { Valor de mercado } \\
\text { del capital accionario } \\
\text { acorde con el modelo } \\
\text { de Opciones Reales Movimiento } \\
\text { Browniano Geométrico }\end{array}$ & $\begin{array}{c}\text { Volatilidad de } \\
\text { los ingresos }\end{array}$ & $\begin{array}{c}\text { Nombre } \\
\text { de la empresa }\end{array}$ & $\begin{array}{c}\text { Valor de mercado } \\
\text { del capital accionario } \\
\text { acorde con el modelo } \\
\text { de Opciones Reales Movimiento } \\
\text { Browniano Geométrico }\end{array}$ & $\begin{array}{c}\text { Volatilidad de } \\
\text { los ingresos }\end{array}$ \\
\hline ALSEA & $37,470,957,917$ & $16.92 \%$ & FEMSA & $994,227,328$ & $12.79 \%$ \\
\hline AMÉRICA MÓVIL & $93,075,895,229$ & $16.76 \%$ & FRAGUA & $25,994,052,482$ & $4.24 \%$ \\
\hline ARA & $1,293,592,359$ & $16.76 \%$ & GENOMMA LAB & $141,923,911$ & $42.30 \%$ \\
\hline ARISTOS & $128,371,794$ & $151.09 \%$ & GIGANTE & $30,189,357,761$ & $25.45 \%$ \\
\hline AUTLAN & $3,376,951,406$ & $53.14 \%$ & GRUPO MÉXICO & $43,177,302,048$ & $17.13 \%$ \\
\hline AXTEL & $7,507,608,314$ & $16.33 \%$ & HERDEZ & $19,329,099,541$ & $81.85 \%$ \\
\hline AZTECA & $4,362,618,080$ & $6.05 \%$ & HOMEX & $735,863,759$ & $184.67 \%$ \\
\hline BACHOCO & $45,872,423,267$ & $20.58 \%$ & HOTELES EXPRESS & $77,891,814,125$ & $9.33 \%$ \\
\hline BENAVIDES & $678,810,400$ & $2.09 \%$ & INTERCERAMIC & $8,189,709,799$ & $9.26 \%$ \\
\hline BIMBO & $190,303,979,599$ & $12.79 \%$ & KIMBERLY & $158,577,865$ & $8.89 \%$ \\
\hline CABLEVISIÓN & $961,469,160,163$ & $35.00 \%$ & KUO & $9,236,069,659$ & $49.01 \%$ \\
\hline CARSO & $163,643,054,884$ & $29.41 \%$ & LALA & $616,450,023$ & $7.41 \%$ \\
\hline CEMEX & $63,079,729,797$ & $27.35 \%$ & LIVERPOOL & $24,258,273,537$ & $78.50 \%$ \\
\hline CHEDRAUI & $36,711,366,994$ & $49.11 \%$ & MÉDICA SUR & $1,857,097,093$ & $12.49 \%$ \\
\hline CIE & $8,443,657,771$ & $53.14 \%$ & MINERA FRISCO & $10,387,152,234$ & $21.92 \%$ \\
\hline COCA COLA FEMSA & $1,450,217,762,654$ & $12.79 \%$ & MOCTEZUMA & $53,596,021,756$ & $12.47 \%$ \\
\hline COLLADO & $1,686,027,262$ & $20.22 \%$ & ROTOPLAS & $9,393,640$ & $21.26 \%$ \\
\hline CRM & $1,764,242,915$ & $16.43 \%$ & URBI & $3,410,303$ & $163.49 \%$ \\
\hline ELEKTRA & $237,795,712,756$ & $17.37 \%$ & VITRO & $24,208,058,890$ & $104.92 \%$ \\
\hline FAMSA & $646,414,884$ & $34.57 \%$ & WALMART & $949,297,174,537$ & $26.40 \%$ \\
\hline
\end{tabular}

Fuente: Elaboración propia

En todos los casos el valor presente expandido es superior al valor obtenido a través del modelo de flujos descontados, lo cual se origina por la flexibilidad que posee la administración de expandir la empresa si la economía está en auge, o bien de contraer la escala de producción si el país se encuentra en recesión. De acuerdo con Trigeorgis (1999) en este análisis se confirma a mayor volatilidad es superior el valor presente expandido, y consecuentemente, el valor de mercado del capital accionario.

En el caso de la elaboración de las Opciones Reales siguiendo un Movimiento Browniano Aritmético, se calcularon los parámetros establecidos en las ecuaciones (31) a (34), bajo el cual se establece que la u es decir

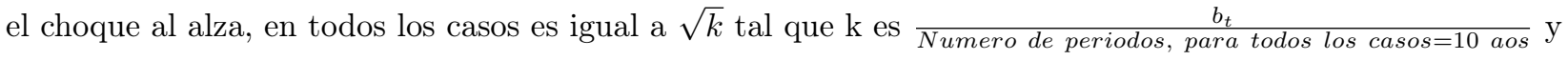

$$
s=b(t)=\int_{0}^{t} e^{-2(r-\delta)} d s=-\frac{1}{2(r-\delta)}\left(1-e^{-2(r-\delta) t}\right)
$$

mientras que la varianza expresada como $\sum_{t}^{2}$ se calculó como la varianza de los ingresos de cada una de las empresas y con ello se pudo despejar la constante

$$
\beta^{2}=\frac{\sum_{t}^{2} 2(r-\delta)}{\left(e^{2(r-\delta) t-1}\right)}
$$

Con lo cual se llegaron a los siguientes valores del mercado del capital accionario comportándose como un movimiento Browniano Aritmético (Tabla 4).

Las probabilidades neutrales al riesgo se fueron calculando para cada nodo del árbol como se muestra a continuación: 


$$
\begin{gathered}
e^{(\boldsymbol{r}-\boldsymbol{\delta}) \boldsymbol{a}(\mathbf{1})}\left(V_{\mathbf{0}}-\beta u\right) \\
\left.q e^{(r-\delta) a(1)}\left(V_{0}+\beta u\right)\right] q+\left[e^{(r-\delta) a(1)}\left(V_{0}-\beta u\right)\right](1-q)=V_{o}(1+r) \\
q=\frac{V_{0}(1+r)\left[e^{(r-\delta) a(1)}\left(V_{0}-\beta u\right)\right]}{\left[e^{(r-\delta) a(1)}\left(V_{0}+\beta u\right)-e^{(r-\delta) a(1)}\left(V_{0}-\beta u\right)\right]}
\end{gathered}
$$

En la Tabla 4 se muestra el valor de mercado del capital accionario, así como la varianza de los flujos calculada con la ecuación (32).

\begin{tabular}{|c|c|c|c|c|c|}
\hline $\begin{array}{c}\text { Nombre } \\
\text { de la empresa }\end{array}$ & $\begin{array}{c}\text { Valor del capital accionario } \\
\text { acorde con el modelo } \\
\text { de Opciones Reales } \\
\text { Movimiento } \\
\text { Browniano Aritmético }\end{array}$ & $\begin{array}{c}\beta^{2} \\
\text { (cifras en miles } \\
\text { de pesos) }\end{array}$ & $\begin{array}{c}\text { Nombre } \\
\text { de la empresa }\end{array}$ & $\begin{array}{c}\text { Valor del capital accionario } \\
\text { acorde con el modelo } \\
\text { de Opciones Reales } \\
\text { Movimiento } \\
\text { Browniano Aritmético }\end{array}$ & $\begin{array}{c}\beta^{2} \\
\text { (cifras en miles } \\
\text { de pesos) }\end{array}$ \\
\hline ALSEA & $37,231,484,673$ & 62,590 & FEMSA & $994,227,328$ & 27,998 \\
\hline AMÉRICA MÓVIL & $95,606,174,117$ & 75,847 & FRAGUA & $25,990,220,910$ & 4,953 \\
\hline ARA & $1,293,592,359$ & 68,262 & GENOMMA LAB & $141,540,754$ & 1,270 \\
\hline ARISTOS & $125,977,061$ & 283 & GIGANTE & $30,151,042,042$ & 3,872 \\
\hline AUTLAN & $3,376,951,406$ & 17 & GRUPO MÉXICO & $43,138,986,329$ & 693 \\
\hline AXTEL & $7,742,071,461$ & 516 & HERDEZ & $19,290,783,822$ & 19,426 \\
\hline AZTECA & $4,324,302,360$ & 1,039 & HOMEX & $735,720,075$ & 5,210 \\
\hline BACHOCO & $45,393,476,778$ & 749 & HOTELES EXPRESS & $77,652,340,881$ & 316 \\
\hline BENAVIDES & $640,494,681$ & 375 & INTERCERAMIC & $8,151,394,080$ & 806 \\
\hline BIMBO & $189,777,138,460$ & 27,998 & KIMBERLY & $149,717,355$ & 2,630 \\
\hline CABLEVISIÓN & $2,534,788,975,996$ & 10 & KUO & $9,232,238,087$ & 1,542 \\
\hline CARSO & $163,604,739,164$ & 5,977 & LALA & $616,066,865$ & 5,532 \\
\hline CEMEX & $63,075,898,225$ & 17,361 & LIVERPOOL & $24,254,441,965$ & 16,569 \\
\hline CHEDRAUI & $33,040,230,294$ & 12,590 & MÉDICA SUR & $1,853,265,521$ & 284 \\
\hline CIE & $8,744,167,055$ & 2,188 & MINERA FRISCO & $10,377,573,305$ & 627 \\
\hline COCA COLA FEMSA & $1,371,378,853,695$ & 16,120 & MOCTEZUMA & $53,592,190,184$ & 1,284 \\
\hline COLLADO & $1,647,711,539$ & 664 & ROTOPLAS & $9,392,682$ & 366 \\
\hline CRM & $1,725,927,196$ & 185 & URBI & $3,314,513$ & 3,302 \\
\hline ELEKTRA & $237,757,397,037$ & 8,669 & VITRO & $24,203,269,425$ & 52 \\
\hline FAMSA & $646,414,884$ & 1,248 & WALMART & $949,258,858,818$ & 51,388 \\
\hline
\end{tabular}

Tabla 4. Valor de mercado del capital accionario calculado con el MBA y la Varianza de los flujos derivada para el modelo. (cifras en pesos)

Se puede observar que el valor de mercado del capital accionario de las empresas bajo el proceso Browniano Aritmético es generalmente inferior, al obtenido a través del proceso Browniano Geométrico.

Una vez calculados los valores de mercado del capital accionario, se dividieron entre el número de acciones en circulación, el cual fue obtenido para cada entidad de la página de la Bolsa Mexicana de Valores en la sección de emisoras. Con ello se puede contrastar cuál modelo es el que brinda la mejor aproximación del valor real cotizado al inicio del mes de abril de 2019; dicho precio se obtuvo de la base de datos de Bloomberg (Tabla 5). 
Tabla 5. Comparación de los precios estimados bajo los tres modelos analizados contra el precio cotizado

\begin{tabular}{|c|c|c|c|c|}
\hline \multirow{2}{*}{$\begin{array}{l}\text { Nombre de } \\
\text { la empresa }\end{array}$} & \multirow{2}{*}{$\begin{array}{l}\text { Precio de } \\
\text { cotización } \\
\text { abril } 2019\end{array}$} & \multicolumn{3}{|c|}{ Precio de la acción } \\
\hline & & $\begin{array}{c}\text { modelo tradicional } \\
\text { de flujos descontados }\end{array}$ & $\begin{array}{c}\text { modelo de Opciones } \\
\text { Reales Movimiento } \\
\text { Browniano Geométrico }\end{array}$ & $\begin{array}{c}\text { modelo de Opciones } \\
\text { Reales Movimiento } \\
\text { Browniano Aritmético }\end{array}$ \\
\hline ALSEA & 44.8500 & 7.7479 & 44.8418 & 44.5552 \\
\hline AMÉRICA MÓVIL & 14.6600 & 9.1157 & 14.0916 & 14.4747 \\
\hline ARA & 2.0800 & 0.0042 & 2.0480 & 2.0447 \\
\hline ARISTOS & 23.7000 & 20.5165 & 24.1773 & 23.7263 \\
\hline AUTLAN & 10.5700 & 8.1740 & 10.0451 & 9.5428 \\
\hline AXTEL & 2.2700 & 2.6215 & 2.7034 & 2.4871 \\
\hline AZTECA & 2.0800 & 0.8239 & 2.0162 & 1.9985 \\
\hline $\mathrm{BACHOCO}$ & 76.8100 & 16.7931 & 76.4540 & 75.6558 \\
\hline BENAVIDES & 13.5000 & 3.0683 & 13.5762 & 12.8099 \\
\hline BIMBO & 40.8200 & 14.2611 & 40.7169 & 40.6042 \\
\hline CABLEVISIÓN & 54.5600 & 10.7995 & 55.0625 & 145.1653 \\
\hline CARSO & 71.9400 & 21.5004 & 71.7280 & 71.7112 \\
\hline CEMEX & 4.8700 & 1.1020 & 4.1671 & 4.1669 \\
\hline CHEDRAUI & 38.2900 & 34.7108 & 38.2463 & 38.2064 \\
\hline CIE & 14.0300 & 1.7205 & 14.0876 & 14.5889 \\
\hline LALA & 26.0000 & 9.9470 & 24.8569 & 24.8414 \\
\hline LIVERPOOL & 122.4000 & 67.5811 & 122.8578 & 122.8384 \\
\hline MINERA FRISCO & 4.1000 & 3.1286 & 4.0808 & 4.0770 \\
\hline ROTOPLAS & 19.7800 & 20.1175 & 20.3207 & 20.3186 \\
\hline VITRO & 50.0000 & 41.2082 & 50.0610 & 50.0511 \\
\hline $\begin{array}{c}\text { COCA COLA } \\
\text { FEMSA }\end{array}$ & 127.2000 & 182.724 & 182.7247 & 172.7911 \\
\hline COLLADO & 8.7600 & 2.3732 & 8.7633 & 8.5642 \\
\hline CRM & 4.0000 & 0.5013 & 5.0309 & 4.9217 \\
\hline ELEKTRA & 1017.6400 & 117.290 & 1018.9125 & 1018.7484 \\
\hline FAMSA & 6.2000 & -1.0761 & 6.3760 & 6.3760 \\
\hline FEMSA & 127.2000 & 28.1311 & 127.6878 & 125.6167 \\
\hline FRAGUA & 256.0000 & 65.6359 & 256.3951 & 256.3573 \\
\hline GENOMMA LAB & 13.9900 & 8.0472 & 13.9141 & 13.8765 \\
\hline GIGANTE & 30.7600 & 23.5437 & 30.3646 & 30.3261 \\
\hline GRUPO MÉXICO & 55.4700 & 32.2918 & 55.4978 & 55.4486 \\
\hline HERDEZ & 44.0500 & 22.9466 & 44.7433 & 44.6546 \\
\hline HOMEX & 0.1350 & -0.0005 & 0.1322 & 0.1322 \\
\hline HOTELES EXPRESS & 20.8900 & 10.1103 & 20.9889 & 20.9243 \\
\hline INTERCERAMIC & 50.0000 & 18.3394 & 50.3474 & 50.1118 \\
\hline KIMBERLY & 123.9000 & 96.1528 & 132.2584 & 124.8685 \\
\hline KUO & 44.7900 & 12.5134 & 44.4149 & 44.3964 \\
\hline MÉDICA SUR & 27.2000 & 5.1917 & 26.6250 & 26.5701 \\
\hline MOCTEZUMA & 60.0000 & 21.1397 & 60.8830 & 60.8786 \\
\hline URBI & 0.0210 & 0.0148 & 0.0221 & 0.0215 \\
\hline WALMART & 54.5600 & 16.6079 & 54.3655 & 54.3633 \\
\hline
\end{tabular}

A continuación, se presenta (Imagen 3), la tasa de descuento, los flujos descontados, el importe de la deuda con costo a valor presente, el número de acciones en circulación, el precio de la acción cotizado a febrero de 2019, así como el comparativo de los árboles binomiales del primero de ellos modelado como un MBG y en el segundo escenario como un MBA para Grupo Alsea. 


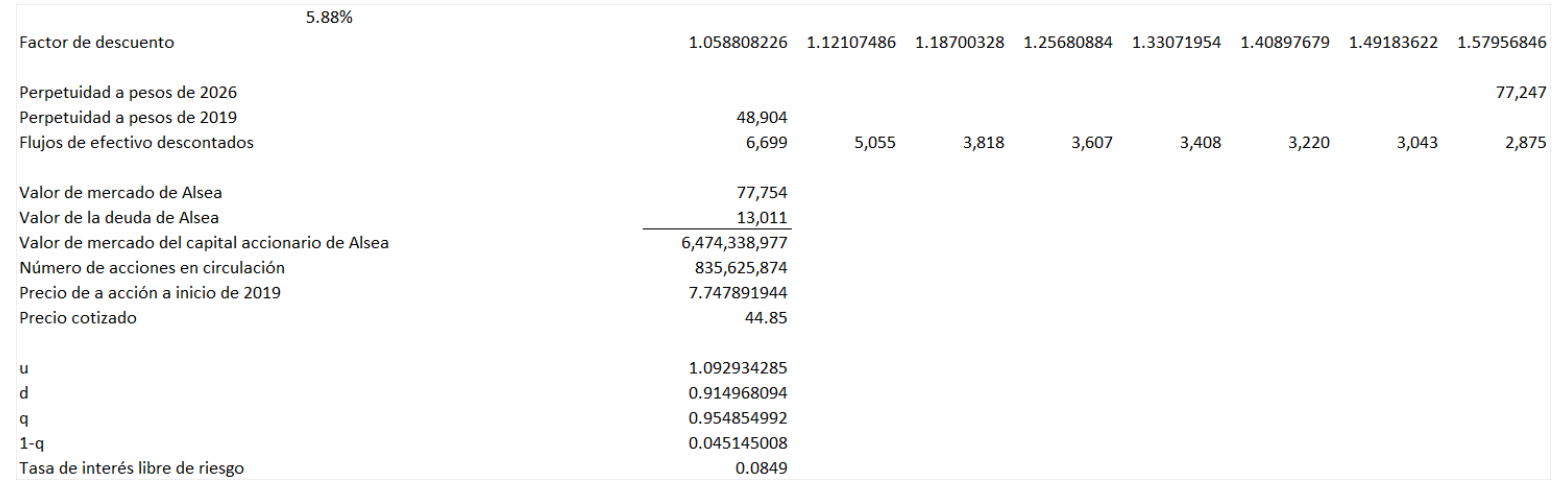

Imagen 3. Resultados del Flujo de Efectivo

Fuente: Elaboración propia

Como se puede observar en la Imagen 3, el valor de mercado de la empresa bajo FED es de $\$ 77,754$ millones de pesos menos el valor presente de la deuda con costo y al dividirlos entre el número de acciones en circulación, se obtiene un precio de $\$ 7.748$; mientras el precio cotizado a inicio del ejercicio de 2019 es $\$ 44.85$, de lo cual se puede derivar que hay una gran variación.

A continuación, se muestra el resultado de las Opciones Reales como un Movimiento Browniano Geométrico (Imagen 4).

\begin{tabular}{|c|c|c|c|c|c|c|c|c|c|}
\hline \multicolumn{9}{|c|}{ Trayectoria del valor de la empresa bajo un Movimiento Browniano Geométrico } & 2027 \\
\hline & 2019 & 2020 & 2021 & 2022 & 2023 & 2024 & 2025 & 2026 & $\begin{array}{r}2027 \\
158,298\end{array}$ \\
\hline & & & & & & & & 144,838 & 132,522 \\
\hline & & & & & & & 132,522 & 121,253 & 110,943 \\
\hline & & & & & & 121,253 & 110,943 & 101,509 & 92,878 \\
\hline & & & & & 110,943 & 101,509 & 92,878 & 84,980 & 77,754 \\
\hline & & & & 101,509 & 92,878 & 84,980 & 77,754 & 71,143 & 65,093 \\
\hline & & & 92,878 & 84,980 & 77,754 & 71,143 & 65,093 & 59,558 & 54,494 \\
\hline & & 84,980 & 77,754 & 71,143 & 65,093 & 59,558 & 54,494 & 49,860 & 45,620 \\
\hline & 77,754 & 71,143 & 65,093 & 59,558 & 54,494 & 49,860 & 45,620 & 41,741 & 38,192 \\
\hline \multicolumn{10}{|l|}{ Opciones reales Movimiento Browniano Geométrico } \\
\hline & 2019 & 2020 & 2021 & 2022 & 2023 & 2024 & 2025 & 2026 & 2027 \\
\hline & & & & & & & & & 789,656 \\
\hline & & & & & & & & 722,477 & 660,260 \\
\hline & & & & & & & 661,012 & 604,083 & 551,933 \\
\hline & & & & & & 604,776 & 552,685 & 504,967 & 461,246 \\
\hline & & & & & 553,324 & 505,661 & 461,998 & 421,991 & 385,326 \\
\hline & & & & 506,249 & 462,637 & 422,685 & 386,077 & 352,527 & 321,768 \\
\hline & & & 463,180 & 423,273 & 386,716 & 353,220 & 322,519 & 294,373 & 268,559 \\
\hline & & 423,774 & 387,259 & 353,809 & 323,158 & 295,066 & 269,311 & 245,689 & 224,014 \\
\hline & 387,720 & 354,309 & 323,701 & 295,655 & 269,950 & 246,382 & 224,766 & 204,932 & 186,723 \\
\hline Valor de la deuda de Alsea & 13,011 & & & & & & & & \\
\hline Valor de mercado del capital accionario de Alsea & $37,470,957,917$ & & & & & & & & \\
\hline Número de acciones en circulación & $835,625,874$ & & & & & & & & \\
\hline Precio de a acción a inicio de 2019 & 44.84178755 & & & & & & & & \\
\hline
\end{tabular}

Imagen 4. Resultados de Opciones Reales Movimiento Browniano Geométrico

Fuente: Elaboración propia

Acorde con este modelo, el valor de mercado de Alsea es de $\$ 37,470,957,917$, considerando las opciones de expansión y contracción, al cual al deducirle la deuda y el flujo de mercado del valor del capital accionario dividido entre el número de acciones en circulación, se llega a que el precio de la acción estimado a inicios del 2019 es de $\$ 44.842$, tendiendo una diferencia porcentual de únicamente -.081\%.

En la Imagen 5 se presentan los resultados de aplicar las Opciones Reales pero modelado el valor de Alsea bajo un proceso Browniano Aritmético. 


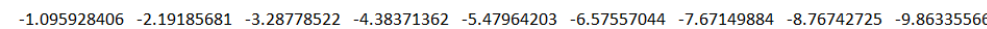

\begin{tabular}{|c|c|c|c|c|c|c|c|c|c|}
\hline & 2019 & 2020 & 2021 & 2022 & 2023 & 2024 & 2025 & 2026 & 2027 \\
\hline & & & & & & & & & $\begin{array}{r}33,881 \\
33,692\end{array}$ \\
\hline & & & & & & & & 37,226 & 33,617 \\
\hline & & & & & & & & 36,978 & 33,730 \\
\hline & & & & & & & 40,810 & 36,895 & 33,579 \\
\hline & & & & & & & 40,583 & 37,019 & 33,768 \\
\hline & & & & & & 44,740 & 40,493 & 36,854 & 33,542 \\
\hline & & & & & & 44,541 & 40,629 & 37,060 & 33,805 \\
\hline & & & & & 49,048 & 44,541 & 40,447 & 36,812 & 33,504 \\
\hline & & & & & 48,884 & 44,391 & 40,674 & 37,102 & 33,843 \\
\hline & & & & 103,184 & 48,775 & 44,591 & 40,402 & 36,771 & 33,466 \\
\hline & & & 95,103 & 53,651 & 48,720 & 44,341 & 40,720 & 37,143 & 33,881 \\
\hline & & 64,624 & 76,959 & 53,531 & 48,939 & 44,341 & 40,356 & 36,730 & 33,391 \\
\hline & 77,754 & 64,479 & 58,750 & 53,531 & 48,775 & 44,441 & 40,493 & 36,895 & 33,617 \\
\hline & 2019 & 2020 & 2021 & 2022 & 2023 & 2024 & 2025 & 2026 & 2027 \\
\hline & & & & & & & & & 165,082 \\
\hline & & & & & & & & & 164,136 \\
\hline & & & & & & & & 181,874 & 163,757 \\
\hline & & & & & & & & 180,628 & 164,325 \\
\hline & & & & & & & 199,869 & 180,213 & 163,568 \\
\hline & & & & & & & 198,729 & 180,836 & 164,514 \\
\hline & & & & & & 219,595 & 198,273 & 180,005 & 163,379 \\
\hline & & & & & & 218,595 & 198,957 & 181,044 & 164,703 \\
\hline & & & & & 241,221 & 218,595 & 198,045 & 179,798 & 163,190 \\
\hline & & & & & 240,397 & 217,844 & 199,185 & 181,251 & 164,893 \\
\hline & & & & 512,984 & 239,848 & 218,845 & 197,811 & $1 / 9,590$ & 163,000 \\
\hline & & & 525,435 & 264,326 & $239,5 / 4$ & 217,594 & 199,413 & 181,459 & 165,082 \\
\hline & 385,326 & 318,685 & 289,927 & 263,724 & 239,848 & 218,094 & 198,273 & 180,213 & $\begin{array}{l}102,022 \\
163,757\end{array}$ \\
\hline Valor de la deuda de Alsea & 13,011 & & & & (2) & 等 & - & (2) & 列 \\
\hline Valor de mercado del capital accionario de Alsea & $37,231,484,673$ & & & & & & & & \\
\hline Número de acciones en circulación & $835,625,874$ & & & & & & & & \\
\hline Precio de a acción a inicio de 2019 & 55520806 & & & & & & & & \\
\hline
\end{tabular}

Imagen 5. Resultados de Opciones Reales Movimiento Browniano Aritmético

Fuente: Elaboración propia

Como se puede desprender al aplicar las Opciones Reales con el MBA, existe una mayor complejidad al determinar el valor de los parámetros necesarios para su desarrollo, y al igual que bajo el MBA el valor del precio de la acción es subestimado en un porcentaje muy pequeño; sin embargo, no brinda en más del $50 \%$ de los casos el mejor ajuste posible. En el ejemplo de Alsea la subestimación es de - $0.66 \%$.

Para que sea más sencillo analizar los resultados, a continuación, en la Imagen 6, se estudiarán las variaciones porcentuales al contrastar los precios cotizados contra los resultantes de cada uno de los modelos.

Como se observa en el Gráfico 1, el modelo que brinda la mayor variación es el de flujos descontados, en la mayor parte de las ocasiones, subestima fuertemente el precio de la acción, excepto en Elektra, Femsa y Fragua (es el valor más alto). Los modelos de Opciones Reales siguiendo los MBG así como los MBA ofrecen resultados muy cercanos a los cotizados, con variaciones pequeñas; no obstante, cabe señalar que brinda una mayor exactitud si se modela como un Movimiento Browniano Geométrico.

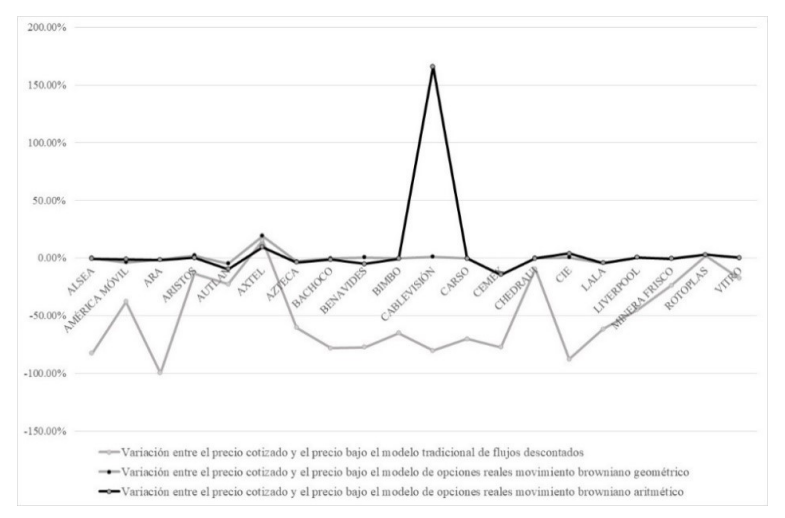




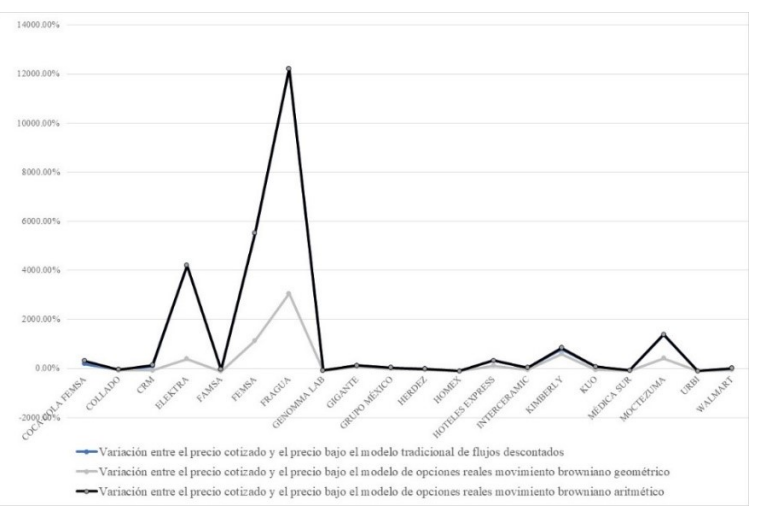

Imagen 6. Análisis comparativo entre el precio de la acción cotizado por empresa y los derivados de los análisis

Fuente: Elaboración propia

En la Imagen 7 se presenta, cuál de estos últimos dos modelos es el que subestima o sobreestima con mayor frecuencia.

\section{Movimiento Browniano Geométrico}

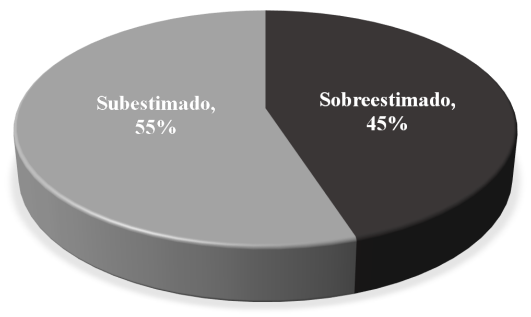

Movimiento Browniano Aritmético

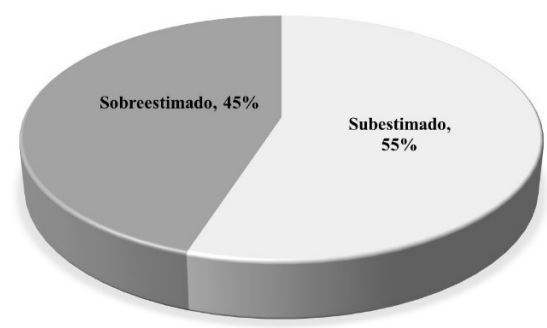

Imagen 7. Análisis de la proporción de subestimación y sobre estimación de las Opciones Reales bajo un MBG y un MBA

Fuente: Elaboración propia

De conformidad con los resultados obtenidos, el MBG subestima con mayor frecuencia los precios de las acciones; en contraste el MBA brinda en la mayoría de las ocasiones una sobreestimación. Asimismo, cabe señalar que a pesar de que los valores ofrecidos por ambos modelos son muy cercanos a los precios cotizados, el MBG ofrece un margen de error inferior al determinado mediante un proceso Browniano Aritmético.

Como se puede observar en la Imagen 8 , las diferencias porcentuales de mayor magnitud, en valor absoluto se presentan al aplicar el MBA en el $58 \%$ de los casos. 


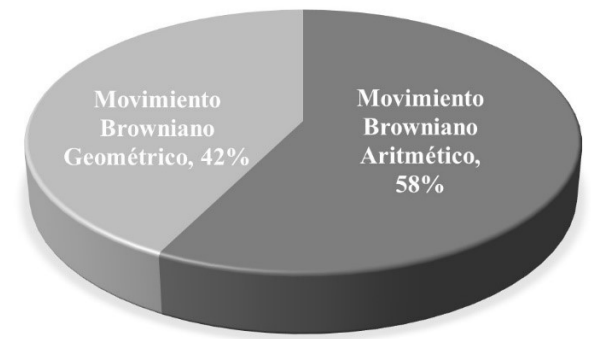

Imagen 8. Modelo que ofrece un margen de error de mayor magnitud Fuente: Elaboración propia

\section{Resultados, Conclusiones y Futuras Líneas de Investigación}

De conformidad con el análisis realizado, a diferencia de lo que dicen Copeland y Antikarov (2001, Capítulo 5) "es mejor el valorar a través de Opciones Reales bajo un proceso Browniano Aditivo" así como por lo determinado por Alexander et al. (2011) quienes afirman que el impacto de la incertidumbre es determinado en mayor medida mediante un proceso aditivo; asimismo aseveran que resulta más sencillo determinar los valores de los parámetros si se utiliza el MBA, lo cual en este trabajo resultó más complejo, tanto en el cálculo de los parámetros, como en la elaboración del árbol binomial que modela la trayectoria del proyecto, brindando como resultado una menor exactitud.

Considerando que el objetivo de este estudio es encontrar el modelo que ofrezca la mejor estimación asequible del Valor Razonable de las partidas que componen los estados financieros, de conformidad con análisis efectuado en este trabajo, los resultados son muy semejantes entre ambos modelos (Opciones Reales bajo los procesos Browniano Geométrico y Browniano Aritmético); sin embargo, el objetivo es que tanto la administración de las empresas, los auditores y demás usuarios apliquen e interpreten el resultado de aquél que brinde el Valor Razonable más exacto posible, derivando en una toma de decisiones más acertada y eficiente tanto de forma interna como externa a la organización. Asimismo, se busca que el modelo propuesto no sea tan complejo en su desarrollo y análisis, es por ello que el proceso matemático del Browniano Geométrico en contraste con el Browniano Aritmético es más sencillo de comprender e implementar.

De igual manera, resulta importante destacar que se efectuó un análisis de correlación entre la volatilidad de los ingresos y el nivel de ajuste de cada modelo, el cual se presenta a continuación.

Correlación entre volatilidad ingresos contra modelo de flujos descontados; 9.59\%. Correlación entre volatilidad ingresos contra modelo de Opciones Reales bajo Movimiento Browniano Geométrico: -7.81\% y correlación entre volatilidad ingresos contra modelo de Opciones Reales bajo Movimiento Browniano Aritmético: $-2.78 \%$.

Como se puede observar, el coeficiente de correlación entre la magnitud de las diferencias entre el precio de la acción cotizado y el precio resultante de la aplicación de cada uno de los modelos no depende en ningún caso de la volatilidad de los ingresos.

Por consecuencia, acorde con los resultados obtenidos se recomienda complementar la valuación a través de flujos de efectivo, con el modelo de Opciones Reales simulando su trayectoria bajo el proceso Browniano Geométrico en sus proyectos de valuación, así como en la valoración de las empresas, del valor de mercado del capital accionario y del precio de la acción, entre otros casos que requieran la determinación de un valor 
justo.

Como se comentó a lo largo del trabajo, existe una gran cantidad de investigaciones, pero muy pocas utilizan otros procesos y no consideran la importancia de modelar las Opciones Reales como un MBA contrastándolo con el tradicional MBG, lo cual le brinda un valor agregado a este trabajo, ya que tampoco existen estudios en esta materia para el caso mexicano. Para futuras investigaciones sería de gran relevancia aplicar y analizar los resultados que propone Climent (2014), ya que propone una solución al sistema de ecuaciones planteado por Cox et al. (1979), cuyo resultado incluye la tasa de interés, la varianza proporcional y el tiempo remanente. La estimación propuesta por este autor tiene diferencias con la estimación de Cox et al. (1979), "la cual es elegante, pero puede subestimar o sobreestimar el modelado del precio del subyacente por la propiedad de tricotomía de los números reales, y como consecuencia subestimar o sobreestimar el riesgo de mercado y también se presentan diferencias en la valuación de las opciones subestimando o sobreestimando el riesgo por la cobertura". En este estudio se consideró una submuestra de únicamente 5 empresas, sobre las cuales se utilizó el modelo de Climent (2014); sin embargo, los resultados de los modelos MBG y MBA son muy cercanos al precio de la acción cotizado al momento de la valuación, mientras que el obtenido bajo la metodología de Climent (2014) sobreestima el precio de la acción en todos los caso en promedio en $15 \%$, pero considerando que se analizó una muestra muy pequeña, será de gran relevancia aplicarlo a una muestra de mayor tamaño.

\section{Referencias}

[1] Alexander, D.; Mo, M. y Stent, A. (2012). Arithmetic Brownian motion and real options. European Journal of Operational Research, 219, (1), 114-122.

[2] Black, F. y Scholes, M. (1973). The Pricing of Options and Corporate Liabilities. Journal of Political Economy, $81,(3), 637-659$.

[3] Bollerslev, J. (1986). Generalized Autoregressive Conditional Heteroscedasty. Journal of Econometrics, 31, (3), 307-27.

[4] Consejo Mexicano de Normas de Información Financieras e Instituto Mexicano de Contadores Públicos. (2018). Normas de Información Financiera. México: Instituto Mexicano de Contadores Públicos.

[5] Copeland, T.E. y Antikarov, V. (2001). Real Options, a Practitioner's Guide. New York: Texere LLC.

[6] Cox, J., S. Ross, and M. Rubinstein. (1979). Option Pricing: A Simplified Approach. Journal o Financial Economics, 7, (3), 229-263.

[7] Dixit, A., y Pindyck, R. (1994). Investment under Uncertainty. New Jersey: Princenton University Press.

[8] Engle, R. F. (1982). Autoregressive Conditional Heteroscedasticity with estimates of the variance of United Kingdom Inflation. Econometrica, 50, (4), 987-1007.

[9] Gamba, A. y Trigeorgis, L. (2007). An Improved Binomial Lattice Method for Multi-Dimensional Options. Applied Mathematical Finance. 14 (5), 453-475.

[10] González-Echeverri, G., Mora-Valencia, A. y Solano, J. (2015). Opciones Reales aplicadas en redes integradas de servicios de salud empleando diferentes métodos de estimación de la volatilidad. Elsevier. Estudios Gerenciales, $31,(136), 287-298$.

[11] Karatzas, I. y Shreve, S.R. (1991). Brownian Motion and Stochastic Calculus. New York: Springer-Verlag.

[12] Lambert, M. y Moreno, M. (2017). Real options valuation under uncertainty. Working Paper.

[13] McDonald, R.L. y Siegel, D. (1986). Value of Waiting to Investment and the Valuation of Firms when There is an Options to Shut Down. The Quarterly Journal of Economics, 101 (4), 331-349. 
[14] Milanesi, G. (2014 [a]). Modelo binomial para la valoración de empresas y los efectos de la deuda: escudo fiscal y liquidación de la firma. Journal of Economics, Finance and Administrative Science, 19, (36), 2-10.

[15] Milanesi, G. (2014 [b]). Valuación probabilística versus borrosa, Opciones Reales y modelo binomial. Aplicación para proyectos de inversión en condiciones de ambigüedad. Elsevier. Estudios Gerenciales, 30, (132), $211-219$.

[16] Ozorio, L., Bastian-Pinto y Teixeira, L. (2013). The Choice of Stochastic Process in Real Option Valuation. Working Paper.

[17] Pliska, S. R. (1998). Introduction to Mathematical Finance, Discrete Time Models. Massachussetts: Blackwell Publishers Inc.

[18] Tresierra, A. y Carrasco C. (2016). Valorización de Opciones Reales: modelo Ornstein-Uhlenbeck. Journal of Economics, Finance and Administrative Science, 21, (41), 56-62

[19] Trigeorgis, L. (1996). Real Options, Managerial Flexibility and Strategy in Resource Allocation. Fourth printing. The MIT Press.

[20] Óksendal, B. (1998). Stochastic Differential Equations: An Introduction with Applications. Fifth Edition. Springer-Verlag. Berlin.

\section{Otras referencias}

[21] Banco de México. (6 de marzo de 2019). Estadísticas. México. Recuperado de https://www.banxico.org.mx/SieInternet.

[22] Damodaran Online Home Page for Aswath Damodaran-NYU Stern. (6 de marzo de 2019). Data. Estados Unidos de Norteamérica. Recuperado pages.stern.nyu.edu/ adamodar/.

[23] Grupo BMV. (6 de marzo de 2019). Emisoras. México. Recuperado https://www.bmv.com.mx. Consultada por última ocasión el 6 de marzo de 2019.

[24] Base de datos de Bloomberg. (6 de marzo de 2019). Estados Unidos de Norteamérica. 